\title{
Unique Conglomeration of Arterial Variations in the Upper Abdomen: CT Angiographic Study
}

\author{
Sehgal $\mathrm{G}^{1}$, Sharma $\mathrm{PK}^{1}$, Kumar $\mathrm{N}^{1}$, Rani $\mathrm{A}^{1}$, Pankaj AK,Parihar $\mathrm{A}^{2}$ \\ ${ }^{1}$ Department ofAnatomy, King George's Medical University, Lucknow-226003, Uttar Pradesh, India. \\ ${ }^{2}$ Department of Radio diagnosis, King George's Medical University, Lucknow-226003,Uttar Pradesh, India
}

\begin{abstract}
Abdominal aortaserves as the main arterial supply for abdominal wall, abdominal organs, lower limbs as well as the male and female genitals. Preoperative computed tomographic angiographyis a precious tool for demonstration of arterial variations and appropriate surgical planning.The present study discusses the presence and clinical significance of vascular variations along with review of literature and comment on embryological development of arterial abnormalities. A clinically and surgically significant, unique combination of vascular variations was diagnosed during computed angiography investigation in a 45 years old male.The celiac trunk was completely absent and all three branches of celiac trunk originated independently from the aorta, this in addition, was accompanied by variations involving other arteries.

The knowledge about abdominal vascular anatomy is very important for operating surgeons and radiologists for the success of upper abdomen surgery as well as imaging interpretation of this region.
\end{abstract}

Keywords: Aorta, variations, celiac trunk, left gastric artery, renal artery, computed tomographic angiography

\section{Introduction}

Abdominal aorta is the continuation of thoracic aorta beyond T12 vertebra up toL4 vertebra, where, it divides into right and left common iliac arteries. It serves as the main arterial supply for all abdominal organs, abdominal wall, lower limbs as well as the male and female genitals. The paired and non-paired branches of abdominal aorta exhibit wide spectrum of variations, well documented in literature [1]. The inferior phrenic arteries are lateral branches of abdominal aorta that help to supply the diaphragm via the abdominal surface. The right and left inferior phrenic arteries arise from the aorta separately but may arise by a common stem or they may originate from the celiac trunk. Occasionally, one may arise from aorta, the other from a renal artery [2]. Although textbook descriptions of the right inferior phrenic artery (Rt IPA) and left inferior phrenic artery (Lt IPA) are typically very brief, they have received increased attention in recent years because of the involvement of the right (most frequently) or left inferior phrenic arteries in the arterial supply and growth of hepatocellular carcinoma [1,2]. The celiac trunk is another branch of abdominal aorta originating from its ventral aspect just below the aortic hiatus. It measures about $1.25 \mathrm{~cm}$ in length and divides into left gastric, common hepatic and splenic artery ("main classic branches" of the celiac trunk). Celiac trunk supplies the foregut and its derivatives including liver, stomach, pancreas and superior part of duodenum. Variations in branching patterns of the celiac trunk like trifurcation, bifurcation and very rarely complete absence have been reported in $1 \%-2 \%$ cases [3].

The aim of the present study is to present an unusual combination of vascular variations in upper abdomen.

\section{Case Report}

A 45-years old male with upper abdominal symptoms was referred to the Department of Radiodiagnosis, King George's Medical University, Lucknow, Uttar Pradesh, India. Angiography was performed on a multislice spiral CT scanner. Two dimensional and three dimensional processed reconstructed images displayed multiple variations.

Celiac trunk was completely absent and all three classic branches of celiac trunk were found originating independently from the aorta. Specifically, left gastric artery (LGA) shared its origin with the right inferior phrenic artery (Rt IPA), as a common gastrophrenic trunk from the anterior aspect of aorta. Splenic artery originated from left anterior aspect of aorta below the origin of gastrophrenic trunk. Common hepatic artery (CHA) originated separately from anterior aspect of aorta towards the right of midline just inferior to the origin of splenic artery. Common hepatic artery divided into three branches (trifurcated) to give rise to gastroduodenal, right hepatic and left hepatic arteries. Double renal arteries were found to be present on the left side (Fig. 1). The left inferior phrenic artery originated independently from the left contour of aorta above the gastrophrenic trunk (Fig. 2A \&2B). An accessory left hepatic artery was present and originated from left gastric artery, it coursed through the gastrohepatic ligament to reach the liver (Fig. 3A \&3B). 


\section{Discussion}

An unusual combination of vascular variations was observed as an incidental finding in a single subject who underwent a CT angiography investigation. Variations involved; the celiac trunk, right inferior phrenic artery, left gastric artery, hepatic artery and left renal artery. Chance discovery of such a combination of variation reaffirms the statement of Jones et al (2001); when there is one vascular variation, there is a higher chance of multiple variations [4].

The celiac trunk is a surgically significant artery originating from the abdominal aorta and supplying the supracolic organs. Branches of this arterial trunk supply the primary organs of abdomen and divert a significant volume of blood from the abdominal aorta. Anatomical variations involving celiac trunk and its branches especially the hepatic and left gastric arteries have been reported by many authors. Absent celiac trunk is a rare variation and has a reported prevalence ranging from $0 \%$ to $2 \%[5,6]$. Preoperative awareness of variations on the part of surgical team is extremely important in order to avoid iatrogenic arterial injury, particularly in the light of increasing number of laparoscopic interventions.

The Right and left IPAs normally arise as lateral branches from the abdominal aorta. In the present case, RtIPA originated from the aorta as a common trunk along with LGA. A CT study by Gokan et al. found most frequent origins of IPA from the aorta more commonly on the right side and from celiac trunk more commonly on the left. Alternative origins from; left gastric, hepatic, superior mesenteric, and spermatic, were also mentioned with $<4 \%$ frequency on either the right or left sides. Potentially, the IPA can communicate with the internal mammary artery, intercostal artery, musculophrenic artery, pericardiophrenic artery and other systemic vessels of the thorax. The right IPA potentially communicates with the intrahepatic arteries. Among the various collateral pathways, one of the most common sources of extrahepatic blood supply to the liver is the right IPA. In cases of occlusion or severe stenosis of hepatic arteries after repeated transcatheter arterial chemoembolization, the hepatic arteries are mainly reconstituted through the right IPA. Therefore, it is important to recognize inferior phrenic arteries during transarterial embolization in patients with hemoptysis [7].

We found an accessory LHA originating from the LGA. Accessory LHAs have been previously reported with a prevalence of $8-15 \%$ [8]. Presence of variant LHA should be acknowledged by surgeons performing lymph node dissection in radical gastrectomy and during laparoscopic treatment of gastroesophageal reflux [9]. The aberrant left hepatic artery (ALHA) coursed through the gastrohepatic ligament to reach the left lobe of the liver. The importance and significance of an ALHA is greatest for liver transplantation and operations performed near the gastrohepatic ligament, including esophago-gastrectomy, gastrectomy, gastric bypass, and anti-reflux procedures [10, 11].

Trifurcation of common hepatic artery into right and left hepatic arteries and gastroduodenal artery with no hepatic artery proper is classified as variant of Type I in Hiatt classification [12]. The knowledge of this variation is important in presurgical planning of liver transplantation, tumour resection, laparoscopic hepatobiliary surgery, hepatic artery infusion chemotherapy and transarterial chemo-embolization [13].

We found double renal arteries supplying the left kidney. Double renal arteries with an aortic origin, represent the persisting embryonic lateral branches of the mesonephros. In a study that included 130 renal angiograms and 32 cadavers, presence of one additional renal artery was seen in $23.2 \%$ and two additional renal arteries in $4.5 \%$. They were seen more commonly on left side $(32 \%)$ as compared to right side $(23.3 \%)$. Presence of one additional renal artery was seen bilaterally in $10.2 \%$ [14]. Failure to acknowledge the presence of these anomalous vessels may result in accidental haemorrhage during urological surgery or renal transplantation inducing segmental ischemia and post-operative hypertension or may increase the complexity of renal transplantation.

The primitive splanchnic arteries located in the dorsal mesentery of the gut, gradually fuse to form the arteries of the foregut, midgut and hindgut as celiac trunk, superior and inferior mesenteric artery respectively. The persistence or unusual development of ventral splanchnic arteries may result in variations of these visceral branches of aorta. The kidneys begin their development in the pelvic cavity but later ascend to their final position in the lumbar region. The pelvic kidneys are supplied by internal iliac artery or common iliac arteries but as they ascend to lumbar region their arterial supply shifts from common iliac artery to abdominal aorta [15]. Lateral mesonephric arteries have been described as arranged in 9 pairs that are divided into cranial, middle and caudal groups in an $18 \mathrm{~mm}$ fetus. Middle group constitutes the 3rd, 4th and 5th pair. This middle group later on persists as renal artery. Persistence of more than one artery from middle group leads to occurrence of accessory renal artery [16].

Most of these asymptomatic vascular variations are identified during the clinical diagnostic investigations or cadaveric studies. Even though presence of these vascular variations is mostly unnoticed, they may be of utmost clinical importance in patients undergoing upper abdominal surgeries, especially transplantation of kidney and liver, surgery for abdominal aortic aneurysms, gastrointestinal surgeries and gonadal surgeries. 


\section{Conclusion}

To our knowledge, multiple vascular variations in a single case, involving an exactly similar combination of variant vasculature, has not been reported in past literatures. We believe that an adequate knowledge of vascular anomalies involving a variety of visceral arteries; in such a complex combination; surely necessitates the need of a presurgical investigation for executing a safe and successful surgical procedure.

\section{Acknowledgements} manuscript.

All authors contributed to conception and design, manuscript preparation, read and approved the final

All authors abide by the Association for Medical Ethics (AME) ethical rules of disclosure.

Competing interests: none to declare

Conflict of interests: none to declare

\section{References}

1. Mcminn RHM. Last's Anatomy, regional and applied, $8^{\text {th }}$ ed. Edinburgh: Churchill Livingstone; 1990.

2. Standring S.Gray's Anatomy- The anatomical basis of clinical practice. $39^{\text {th }}$ ed. New York: Elsevier/Churchill Livingstone; 2008. p 1116-1117.

3. Petrella S, Rodrigues CFS, Sgrott EA, Fernandez GJM, Marques SR. \& Prates JC. Anatomy and variations of the celiac trunk. Int. J. Morphol. 2007; 25(2): 249-257

4. Jones RM, Hardy KJ. The Hepatic artery: a reminder of surgical anatomy. J R Coll. Surg. Edinb. 2001; 46: 168-170.

5. Chen H, Yano R, Emura S, Shoumura S. Anatomic variation of the celiac trunk with special reference to hepatic artery patterns. Ann Anat. 2009; 191: 399-407.

6. Lipshutz B. A composite study of the coeliac axis artery. Ann. Surg. 1917; 65:159-169

7. Gokan T, Hashimoto T, Matsui S, Kushihashi T, Nobusawa H, Munechika H. Helical CT demonstration of dilated right inferior phrenic arteries as extrahepatic collateral arteries of hepatocellular carcinomas. J. Comput. Assist. Tomogr. 2001; 25:68-73.

8. Covey AM, Brody LA, Maluccio MA. Variant hepatic arterial anatomy revisited: Digital subtraction angiography performed in 600 patients. Radiology. 2002; 224: 542-547.

9. Hendrickson RJ, Yu S, Bensard DD, Petty JK, Patrick DA, Karrer FM. Preservation of an aberrant left hepatic artery during laparoscopic Nissen fundoplication. JSLS. 2006; 10(2): 180-183.

10. Klingler PJ, Seelig MH, Floch NR, et al. Aberrant left hepatic artery in laparoscopic antireflux procedures. Surg. Endosc. 2004; 18 (5): 807-811.

11. Okano S, Sawai K, Taniguchi H, et al. Aberrant left hepatic artery arising from the left gastric artery and liver function after radical gastrectomy for gastric cancer. World J. Surg. 1993; 17 (1): 70- 73.

12. Hiatt JR, Gabbay J, Busutil RW. Surgical anatomy of the hepatic arteries in 1000 cases. Ann. Surg. 1994; $220: 50-2$.

13. Lee KH, Sung KB, Lee DY, Park SJ, Kim KW, Yu JS. Transcatheter arterial chemoembolization for hepatocellular carcinoma: anatomic and hemodynamic considerations in the hepatic artery and portal vein. Radiographics. 2002; 22 (5): 1077 - 1091.

14. Satyapal KS, Haffejee AA, Singh B, Ramsaroop L, Robbs JV, Kalideen JM. Additional renal arteries incidence and morphometry. Surg. Radiol. Anat. 2000; 23(1):33-38.

15. Sadler TW. Digestive System. Langman's Medical Embryology. $8^{\text {th }}$ ed. Philadelphia: Williams \& Wilkins; 2000, p 270-30.

16. Keibel F, Mall FP (eds), Manual of human embryology, Vol. 2, J.B. Lippincott, Philadelphia, 1912, p 820-825.

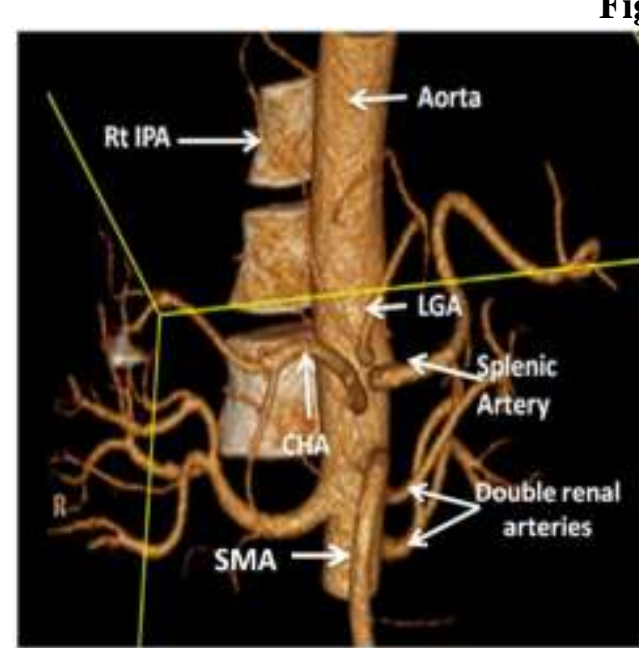

Fig.1. Volume rendered Three dimensional (3D) image showing absent celiac trunk. The three classic branches are seen originating separately from the aorta. Also visualized are the common gastrophrenic trunk (LGA \& Rt IPA) and double renal arteries on left side. (LGA- Left gastric artery, Rt IPA- Right inferior phrenic artery, CHA- Common hepatic artery, SMA- Superior mesenteric artery)
Figures:
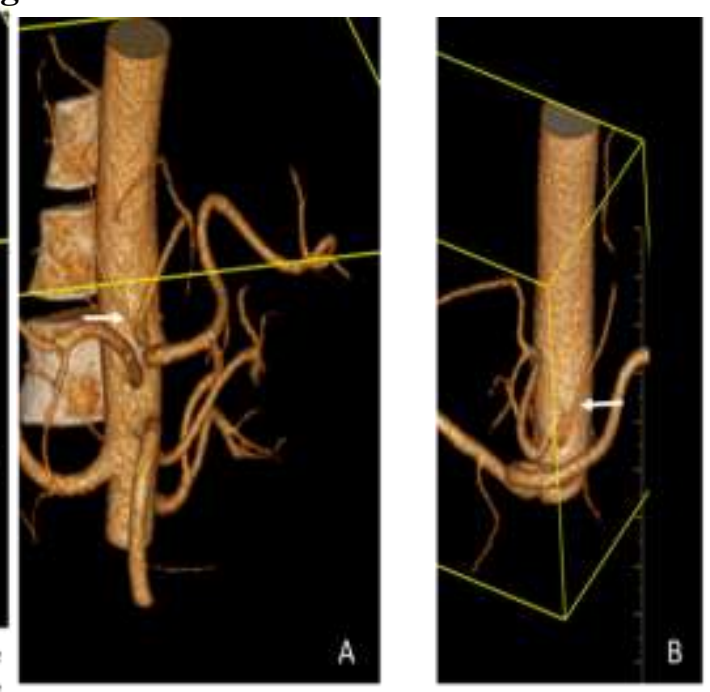

Fig 2. Volume rendered 3D images Fig 2- A: showing origin of Right Inferior phrenic artery and Left gastric artery from a common trunk (arrow) \& Fig.2- B: showing the origin of Left inferior phrenic artery from aorta (arrow) 

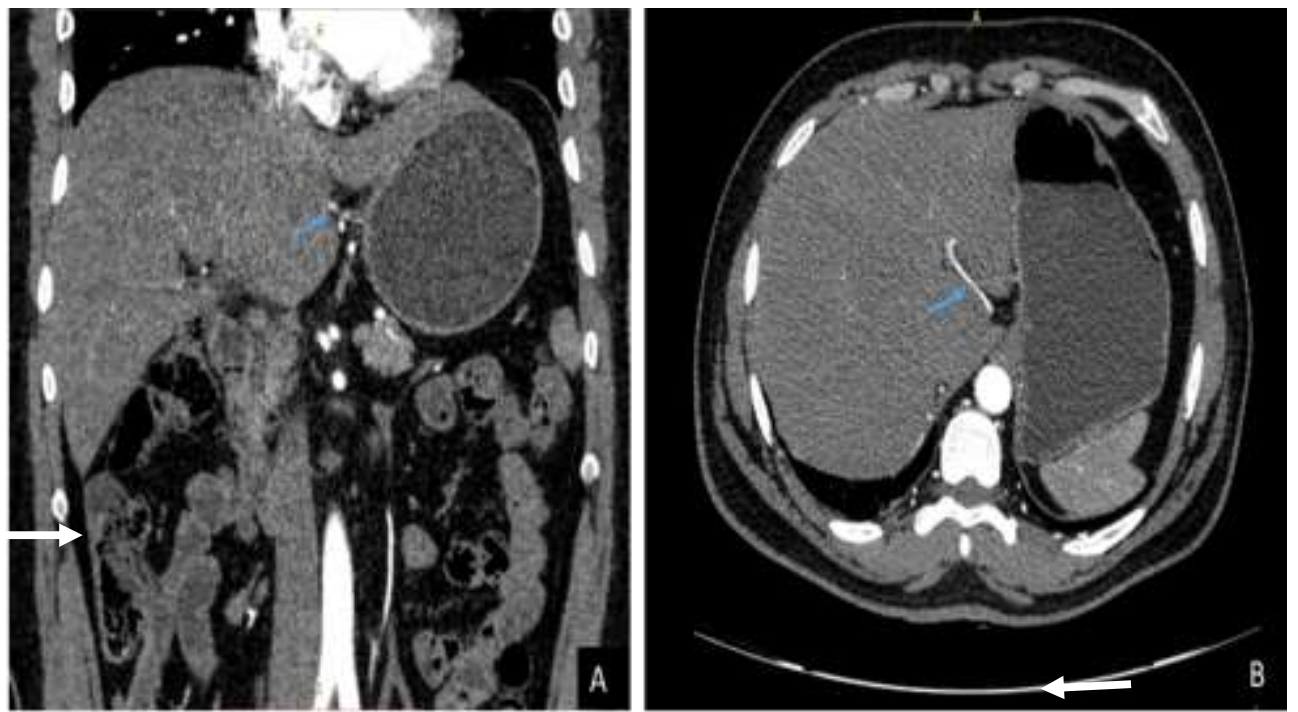

Fig. 3A: Coronal Multiplanar reconstruction showing origin of accessory LHA from Left Gastric Artery (arrow) \& Fig. 3B: Axial image showing the course of aoc. LHA through gastrohepatic ligament (arrow) 